\title{
X Congreso Ibérico de Agroingeniería

\section{Análisis del aclareo mecánico de mandarinas durante el desarrollo del fruto a través de los videos de desprendimiento en laboratorio}

\author{
Angelo Romano', Antonio Torregrosa' ${ }^{2}$, Coral Ortiz ${ }^{2}$ \\ 1 Universidad de Firenza, Italia \\ 2 Universitat Politècnica de València, Dpto. Ingeniería Rural y Agroalimentaria, Cno. Vera s/n, 46022 \\ Valencia; cortiz@dmta.upv.es
}

\begin{abstract}
Resumen: La operación de aclareo consiste en la eliminación de parte de los frutos de un árbol antes del desarrollo con la finalidad de incrementar los calibres de los frutos en recolección. El aclareo mecánico se ha abordado en otros frutales, pero en cítricos se realiza de forma manual en ciertas variedades. Basándonos en resultados de estudios previos, el objetivo del presente trabajo fue estudiar el aclareo mecánico de cítricos a lo largo del desarrollo del fruto. Para ello se usó un equipo de vibrado de ramas en laboratorio y una cámara de alta velocidad para evaluar el desprendimiento de los frutos a lo largo del tiempo. El análisis de las imágenes de la vibración muestra el porcentaje de derribo evoluciona de forma acdente frente al tiempo ajustándose a una curva inversa de $\mathrm{X}$ o de tipo sigmoidal. Para poder conseguir un porcentaje de derribo aceptable se podría vibrar 5 segundos cuando los frutos tienen un calibre mayor de $20 \mathrm{~mm}$, un tiempo mayor de $8 \mathrm{~s}$ para calibres de $14 \mathrm{~mm}$ y más de $10 \mathrm{~s}$ para calibres de $8 \mathrm{~mm}$ (bajo las considiciones de laboratorio del estudio).
\end{abstract}

Palabras clave: aclareo, mandarina, desprendimiento, video

\section{Introducción}

La operación de aclareo es la eliminación de flores o frutos de los árboles antes de la fecha de recolección para mejorar la producción y la calidad de los frutos (Ouma, 2012).

Existen diferentes métodos para aclarar: químicos, manuales y mecánicos. El aclareo manual representa un coste muy elevado [1].

Son muchos los trabajos realizados sobre aclareo químico ([2], [3]). Sin embargo, estos trabajos se ven reducidos por la limitación del uso de algunos productos [4].

El aclareo mecánico ha sido ensayado en melocotones con vibradores de troncos y vibradores electromagnéticos ([5], [6]). Además en algunos frutales de hueso y pomáceas se han estudiado equipos de vibrado para el aclareo mecánico de flores y frutos ([7], [8], [9]). Estos estudios demuestran que el aclareo mecánico reduce tiempo y costes de cultivo.

En cítricos el aclareo de frutos no ha sido muy estudiado. [10] estudiaron el aclareo mecánico en dos variedades de mandarinas ('Clemenrubí' y 'Clemenules') con un peine eléctrico y un vibrador de ramas de gasolina. Se consiguieron porcentajes de derribo entre $31 \%$ y $57 \%$. [11] realizando ensayos de campo con vibradores de ramas determinaron que el aclareo mecánico necesita más de 30 días para que los frutos de los árboles aclareados puedan tener un tamaño significativamente mayor que los frutos de los árboles no aclareados. 


\section{CONGRESO IBÉRICO DE AGROINGENIERÍA \\ X CONGRESSO IBÉRICO DE AGROENGENHARIA \\ 3 - 6 septiembre 2019, Huesca - España}

Actualmente en la Comunidad Valenciana, el aclareo manual de mandarinos es una operación habitual en ciertas variedades. Estas operaciones manuales se realizan en repetidas tandas y encarecen los costes de cultivos. Por ello, se ha considerado la necesidad de estudiar el aclareo de mandarinos.

Para conseguir la máxima eficiencia en las prácticas agrícolas, estas deben realizarse en el momento de desarrollo vegetativo adecuado [12]. En el caso del aclareo de mandarinos elegir el momento de desarrollo del fruto adecuado es crucial.

En el presente trabajo se abordó el estudio en laboratorio de vibrado mecánico de ramas de mandarino para aclareo durante el desarrollo fenológico de los frutos de mandarino.

\section{Materiales y métodos}

\subsection{Equipo de vibrado}

Se utilizó un equipo de vibrado unidireccional de ramas (Figura 1). El movimiento rotatorio de accionamiento eléctrico es transformado en un movimiento lineal. Un convertidor de frecuencia se utiliza para variar la frecuencia de vibrado. La amplitud se puede fijar en un rango de $0,01 \mathrm{~m}$ a $0,180 \mathrm{~m}$, fijando el vástago en los distintos orificios (A). La rama se coloca en una posición vertical hacia abajo sujeta por una abrazadera (B).

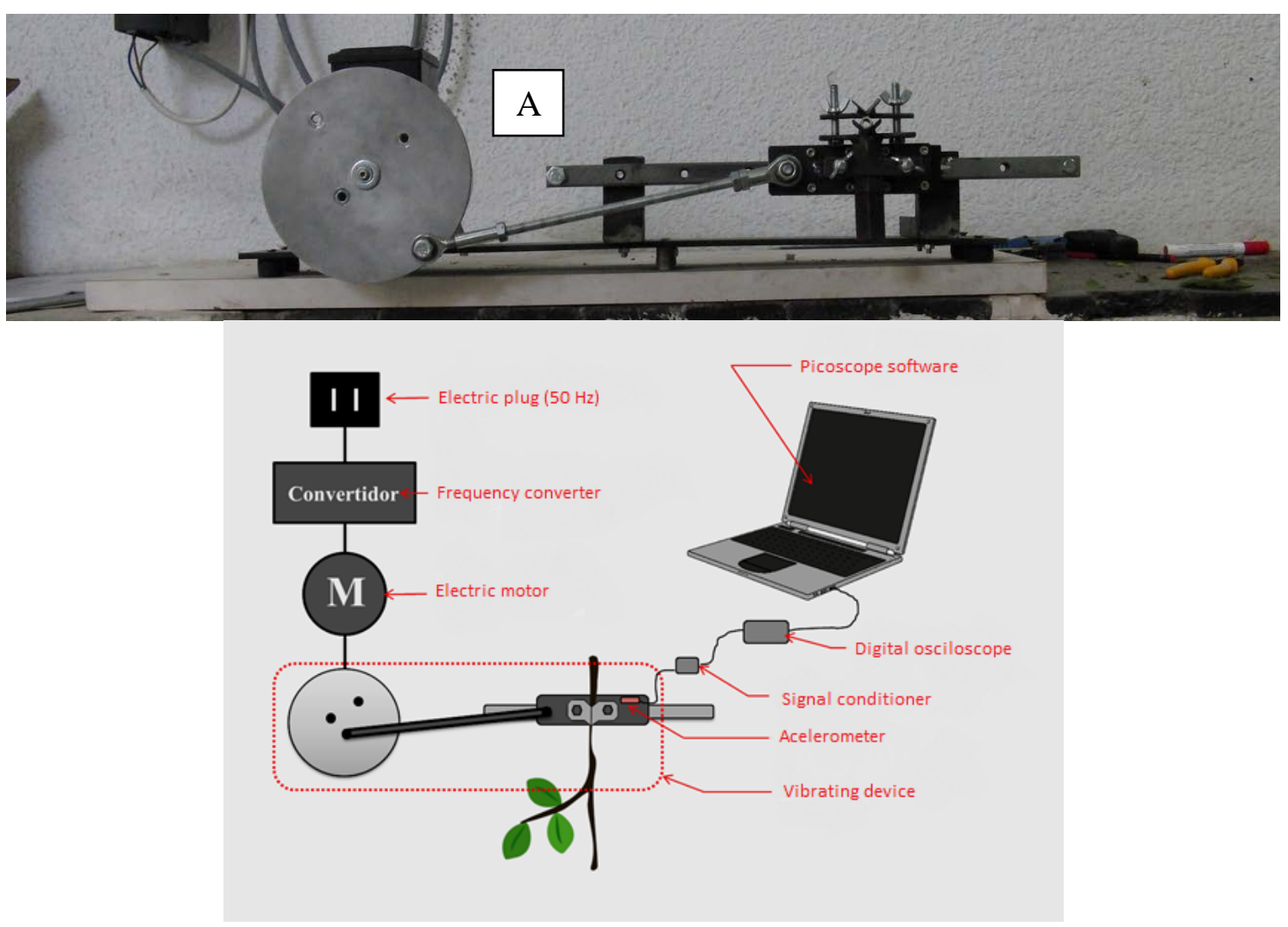

Figura 1. Dispositivo de vibrado de laboratorio.

Una cámara de alta velocidad (300 fotogramas/s) (Casio EXF1, www.exilim.com) se utilizó para grabar el movimiento de la rama y registrar la caída de los frutos.

Las frecuencias reales se midieron con un acelerómetro (Kistler 8763A500) y con las grabaciones de alta velocidad. 


\section{CONGRESO IBÉRICO DE AGROINGENIERÍA \\ X CONGRESSO IBÉRICO DE AGROENGENHARIA \\ 3 - 6 septiembre 2019, Huesca - España}

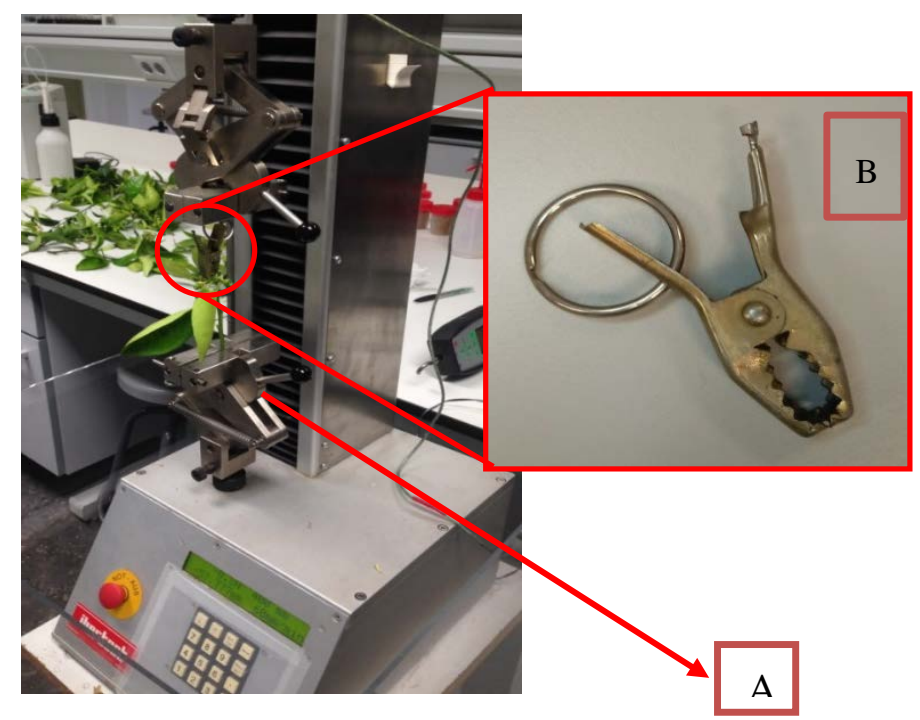

Figura 2. Máquina Universal de Ensayos utilizada en el test de tracción para obtener fuerza de retención.

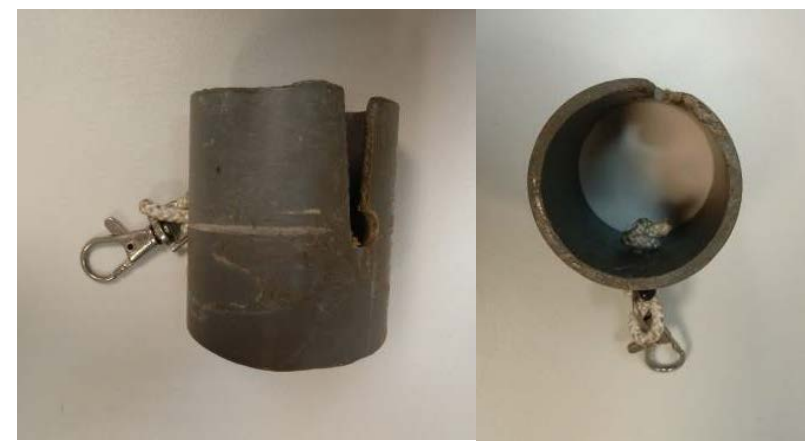

Figura 3. Dispositivo para el agarre de los rutos verdes en el ensayo de tracción .

Además, se realizó la medida de la fuerza de retención mediante un ensayo de tracción con una máquina universal de ensayos (Ibertest, www.ibertestint.com), figura 2. La rama se colocaba hacia abajo sujeta mediante una agarradera (figura 2A). Como vástago de tracción se utilizó una pinza para los frutos más pequeños (figura $2 \mathrm{~B}$ ) y un cilindro con ranura para los frutos mayores (figura 3). Una muestra de 20 frutos por semana era utilizada para el ensayo de fuerza de retención. El peso y calibre de esta muestra de frutos se medía también y se ampliaba la medida de calibre con 100 frutos más.

\subsection{Material vegetal}

Para el ensayo definitivo se utilizaron ramas de mandarinos de la variedad 'Clemenrubí' de una plantación situada en el Racó de Gaussa (Sagunto), sobre la que también se realizó un ensayo de aclareo en campo. Durante 7 semanas se seleccionaron cad semana 20 ramas de forma similar con un número de frutos entre 3 y 20.

\subsection{Metodología}

Basándonos en los resultados de los ensayos preliminares, para el ensayo definitivo se estableció una carrera de 0,03 m. Las frecuencias estudiadas $18,1 \mathrm{~Hz}$ y $38,6 \mathrm{~Hz}$. 


\section{CONGRESO IBÉRICO DE AGROINGENIERÍA \\ X CONGRESSO IBÉRICO DE AGROENGENHARIA \\ 3 - 6 septiembre 2019, Huesca - España}

Siguiendo la metodología empleada previamente por [13], las películas de la caída de los frutos se utilizaron para calcular los tiempos y porcentajes de desprendimiento de los frutos, mediante la ecuación [1].

$$
t_{i j}=\frac{\left(\text { foto }_{d}-\text { foto }_{0}\right)}{300}
$$

$$
\begin{aligned}
& \text { tij= tiempo (s) de la fruta i de la rama j. } \\
& \text { photo } 0 \text { fotograma cuando empieza el movimiento. } \\
& \text { photo d, fotograma cuando se desprende el fruto i. }
\end{aligned}
$$

Para poder determinar el porcentaje de desprendimiento en relación con el tiempo de vibrado, el número acumulado de frutos desprendido respecto del total fue calculado agrupando las ramas vibradas bajo la misma frecuencia (simulando un árbol con todas las ramas con las mismas condiciones), ecuación [2].

$$
\% D(t)=\frac{\Sigma \text { frutos desprendidos }(t)}{\text { Número total de frutos }}
$$

\subsection{Metodología estadística}

Se ha evaluado el efecto de la frecuencia y la semana (estado de desarrollo del fruto) en el porcentaje de derribo mediante un análisis de varianza multifactorial. Además, se han estudiado las curvas de ajuste de la evolución del porcentaje de derribo acumulado a lo largo del tiempo, para cada frecuencia de vibrado y semana.

\section{Resultados y discusión}

\subsection{Evolución de los parámetros calibre, masa y fuerza de retención}

En un primer paso se estudió la evolución de los parámetros, peso, calibre, fuerza de tracción y porcentaje de desprendimiento (para cada frecuencia) a lo largo de las semans de ensayo, figura 4.

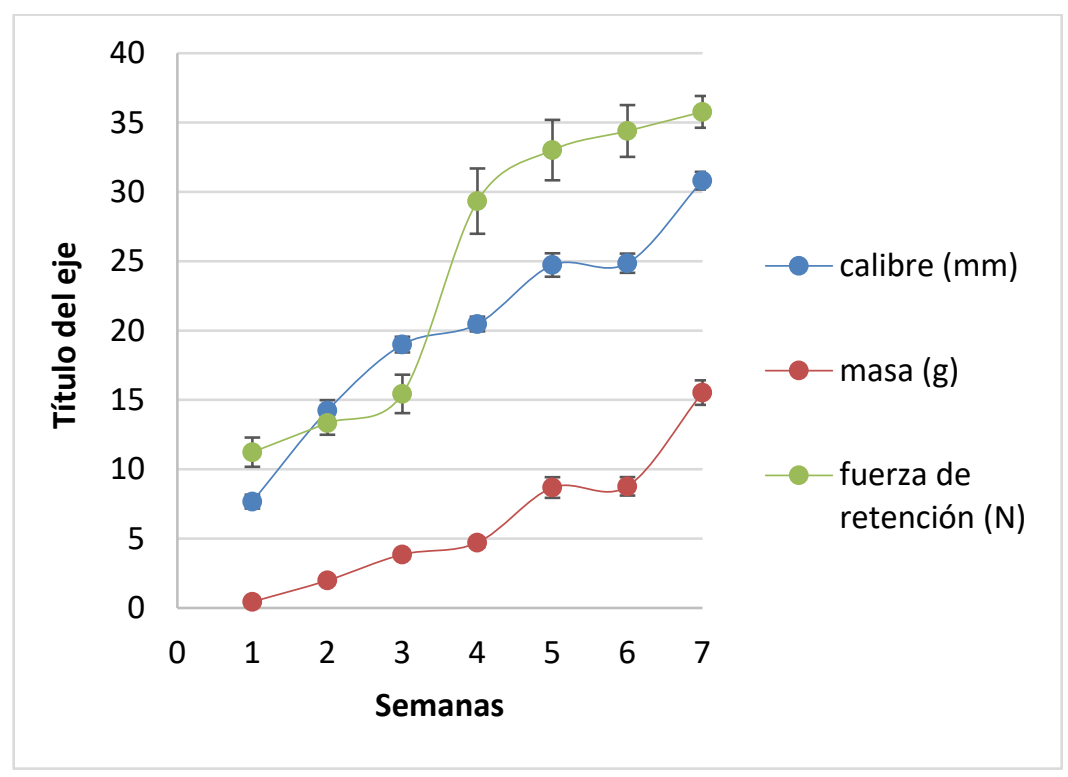

Figura 4. Evolución de la masa, calibre y fuerza de retención a lo largo de las semana (medias y errores típicos). 


\section{CONGRESO IBÉRICO DE AGROINGENIERÍA \\ X CONGRESSO IBÉRICO DE AGROENGENHARIA \\ 3 - 6 septiembre 2019, Huesca - España}

El análisis de varianza mostró que el factor Semana afecta de forma significativa a los parámetros de calibre, masa y fuerza de retención (tablas 1, 2 y 3).

Tabla 1. Análisis de varianza (suma de cuadrados tipo II) del efecto del factor semana en el calibre de los frutos.

\begin{tabular}{cccccc}
\hline Fuente & Suma de Cuadrados & Gl & Cuadrado Medio & Razón-F & Valor-P \\
\hline Entre grupos & 4378,33 & 5 & 875,665 & 100,53 & 0,0000 \\
Intra grupos & 992,988 & 114 & 8,71042 & & \\
Total (Corr.) & 5371,31 & 119 & & & \\
\hline
\end{tabular}

Tabla 2. Análisis de varianza (suma de cuadrados tipo II) del efecto del factor semana en la masa de los frutos.

\begin{tabular}{cccccc}
\hline Fuente & Suma de Cuadrados & G1 & Cuadrado Medio & Razón-F & Valor-P \\
\hline Entre grupos & 1172,58 & 5 & 234,516 & 55,69 & 0,0000 \\
Intra grupos & 480,047 & 114 & 4,21094 & & \\
Total (Corr.) & 1652,63 & 119 & & & \\
\hline
\end{tabular}

Tabla 3. Análisis de varianza (suma de cuadrados tipo II) del efecto del factor semana en la fuerza de retención de los frutos.

\begin{tabular}{cccccc}
\hline Fuente & Suma de Cuadrados & G1 & Cuadrado Medio & Razón-F & Valor-P \\
\hline Entre grupos & 7669,93 & 5 & 1533,99 & 26,26 & 0,0000 \\
Intra grupos & 6659,94 & 114 & 58,4205 & & \\
Total (Corr.) & 14329,9 & 119 & & & \\
\hline
\end{tabular}

Sin embargo, aunque se observa una evolución creciente de los parámetros a lo largo de las semanas, el test de rangos múltiples de Duncan mostró que no existían diferencias en calibre, peso y fuerza de retención entre algunas semanas contiguas (tablas 4, 5 y 6). Las semanas 3 y 4 y las semanas 5 y 6 presentaban valores de calibre y masa similares. En el caso de la fuerza de retención, parece haber un cambio entre las semanas 1,2 y 3 y las semanas 4, 5, 6 y 7. Observando la evolución de la fuerza de retención (figura 4) se observa una tendencia creciente. Los datos de fuerza de retención registrados las semanas 3 y 6 presentan valores menores que la tendencia creciente de la curva. Esto puede ser un error causado por tomar la medida al finalizar el ensayo de desprendimiento con el vibrador de laboratorio.

Pruebas de Múltiple Rangos para calibre (mm) por 2018 semana

Tabla 4. Prueba de rangos múltiples de Duncan del efecto de la semana en el calibre de los frutos.

\begin{tabular}{ccccc}
\hline Semana & Casos & Media & \multicolumn{2}{c}{ Grupos Homogéneos } \\
\hline 1 & 20 & 7,669 & $\mathrm{X}$ & \\
2 & 20 & 14,2285 & $\mathrm{X}$ & \\
3 & 20 & 18,9905 & $\mathrm{X}$ & \\
4 & 20 & 20,472 & $\mathrm{X}$ & \\
5 & 20 & 24,7285 & $\mathrm{X}$ & \\
6 & 20 & 24,859 & $\mathrm{X}$ & \\
7 & 20 & 30,81 & $\mathrm{X}$ & \\
\hline
\end{tabular}


X CONGRESO IBÉRICO DE AGROINGENIERÍA

X CONGRESSO IBÉRICO DE AGROENGENHARIA

3- 6 septiembre 2019, Huesca - España

Tabla 5. Prueba de rangos múltiples de Duncan del efecto de la semana en la masa de los frutos.

\begin{tabular}{cccc}
\hline Semana & Casos & Media & Grupos Homogéneos \\
\hline 1 & 20 & 0,4464 & $\mathrm{X}$ \\
2 & 20 & 1,98395 & $\mathrm{X}$ \\
3 & 20 & 3,85565 & $\mathrm{X}$ \\
4 & 20 & 4,71145 & $\mathrm{X}$ \\
5 & 20 & 8,68445 & $\mathrm{X}$ \\
6 & 20 & 8,77195 & $\mathrm{X}$ \\
7 & 20 & 15,5299 & $\mathrm{X}$ \\
\hline
\end{tabular}

Tabla 6. Prueba de rangos múltiples de Duncan del efecto de la semana en la fuerza de retención de los frutos.

\begin{tabular}{cccc}
\hline Semana & Casos & Media & Grupos Homogéneos \\
\hline 1 & 20 & 11,235 & $\mathrm{X}$ \\
3 & 20 & 15,435 & $\mathrm{XX}$ \\
2 & 20 & 16,01 & $\mathrm{X}$ \\
6 & 20 & 25,71 & $\mathrm{X}$ \\
4 & 20 & 29,335 & $\mathrm{XX}$ \\
5 & 20 & 33,015 & $\mathrm{XX}$ \\
7 & 20 & 35,77 & $\mathrm{X}$ \\
\hline
\end{tabular}

Además, se evaluó la evolución del parámetro fuerza de retención entre masa, figura 5.

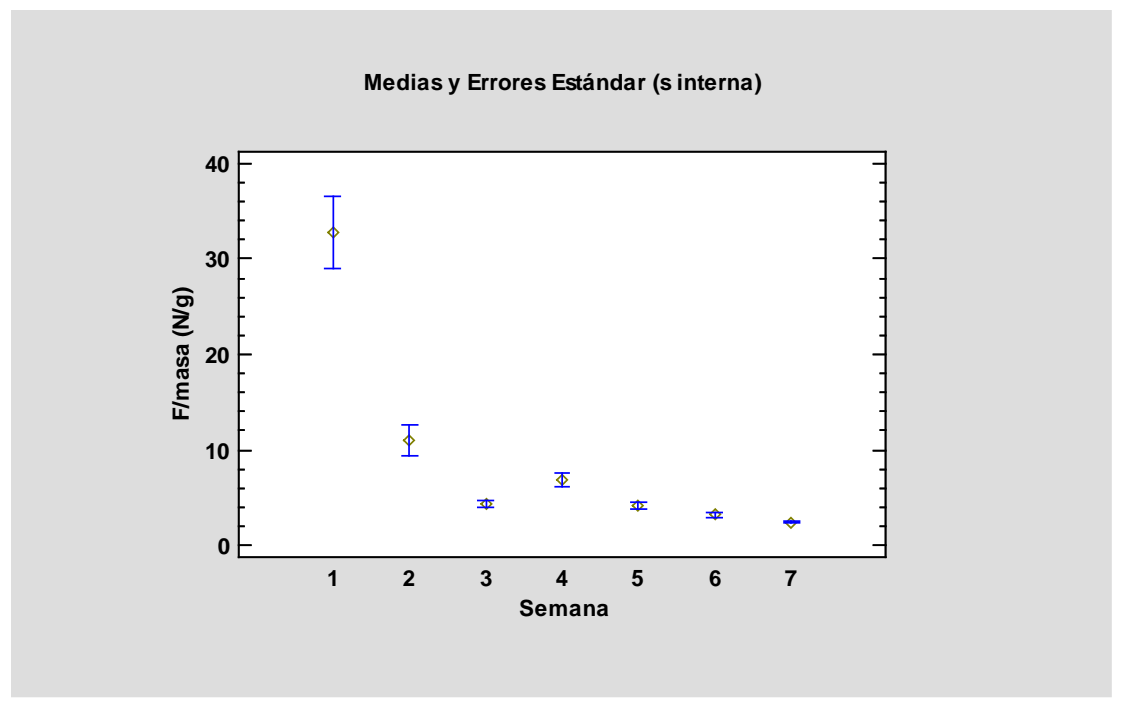

Figura 5. Evolución del ratio fuerza de retención entre masa (N/g) a lo largo de las semanas

Para evaluar el desarrollo de los frutos a lo largo de las semanas de ensayo se utilizaron las curvas de crecimiento de los frutos de las campañas 2017 y 2018 (ecuaciones [1] y [2]) que se han obtenido en los trabajos paralelos de campo sobre aclareo.

Para el año 2017: calibre $=30,0+0,336 \times$ día [1]

Para el año 2018: calibre $=25,7+0,307 \times$ día [2]

Estas curvas para los frutos en estado fenológico 72 (según la escala BBCH de [14]) y los resultados de los test de rangos múltiples de Duncan de calibre y masa confirmaron que las muestras analizadas cada semana no siempre correspondían con estados de desarrollo con la misma pauta de evolución. 


\section{CONGRESO IBÉRICO DE AGROINGENIERÍA \\ X CONGRESSO IBÉRICO DE AGROENGENHARIA \\ 3 - 6 septiembre 2019, Huesca - España}

Por ello se procedió a corregir las semanas por estadios relacionados con el nivel de desarrollo de los frutos. El estadio 1 corresponde con la semana 1, el estadio 2 con la 2, el estadio 3 con las semanas 3 y 4 , el estadio 4 con las semanas 5 y 6 , y el estadio 5 con la semana 7 . En la tabla 7 y figura 6 se presenta el test de Duncan de diferencias significativas entre los calibres de los 5 estadios y los valores medios de calibre correspondientes a cada estadio.

Tabla 7. Prueba de rangos múltiples de Duncan del efecto del estadio en el calibre de los frutos.

\begin{tabular}{cccc}
\hline Estadio & Casos & Media & Grupos Homogéneos \\
\hline 1 & 20 & 7,669 & $\mathrm{X}$ \\
2 & 20 & 14,2285 & $\mathrm{X}$ \\
3 & 40 & 19,7313 & $\mathrm{X}$ \\
4 & 40 & 24,7937 & $\mathrm{X}$ \\
5 & 20 & 30,81 & $\mathrm{X}$ \\
\hline
\end{tabular}

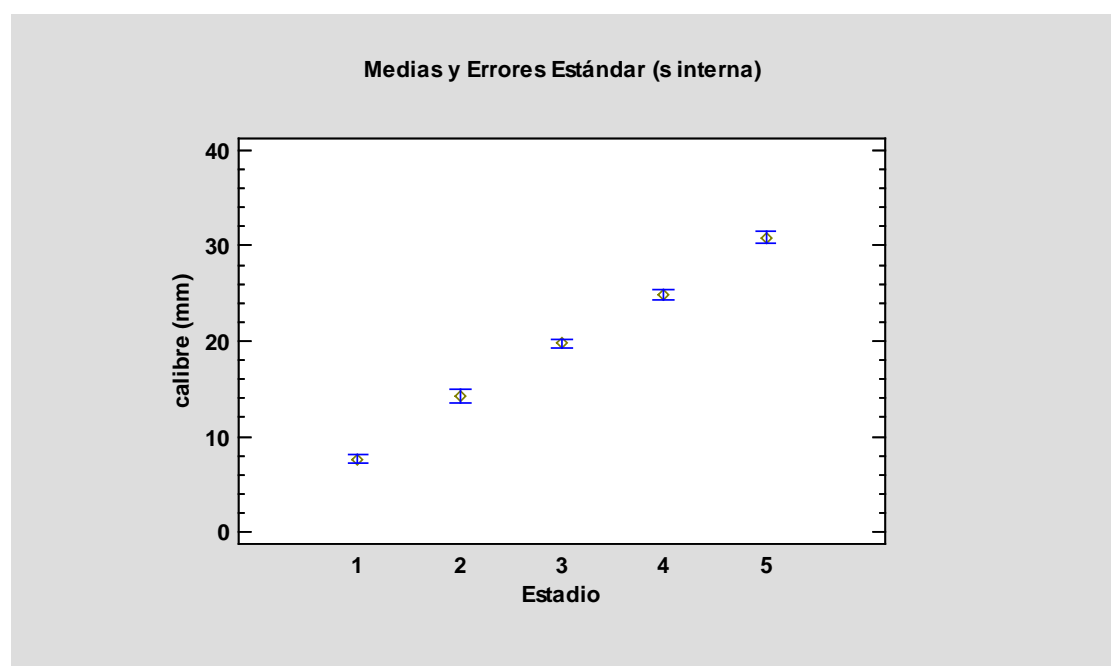

Figura 6. Evolución del calibre (mm) según los estadios.

Con esta corrección la evolución del ratio fuerza de retención entre masa presenta un rápido descenso en los estadios 1, 2 y 3 mientras que los estadios 3,4 y 5 se mantienen similares, figura 6 y tabla 8.

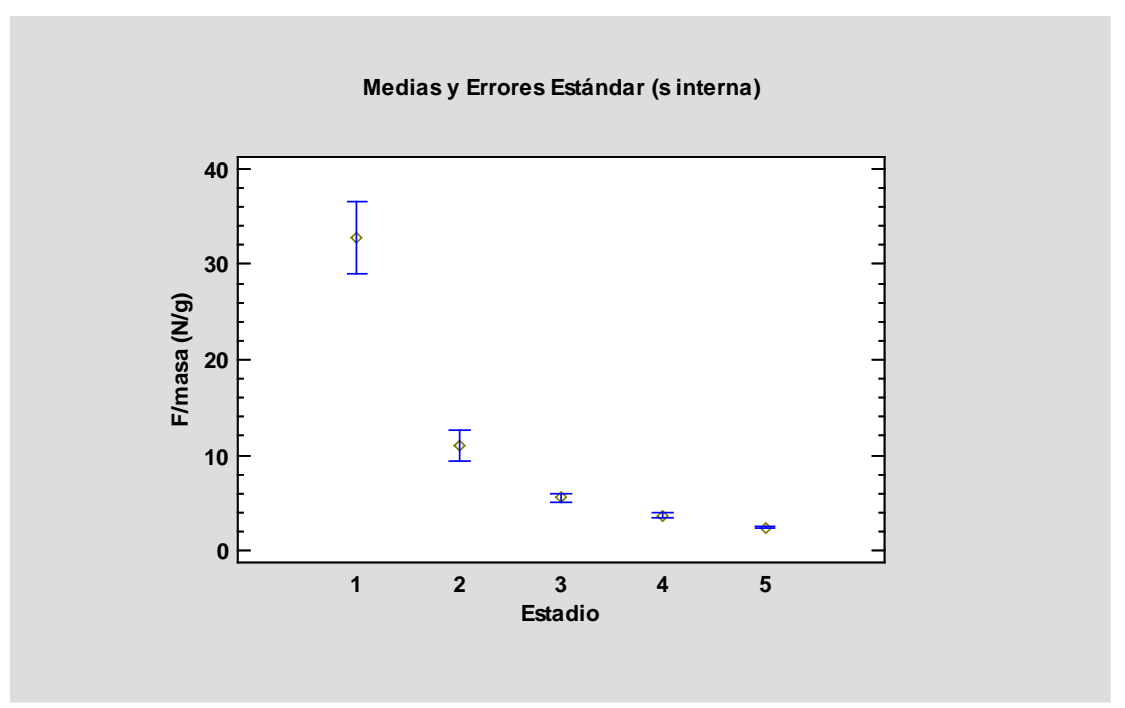

Figura 7. Evolución del ratio fuerza de retención entre masa $(\mathrm{N} / \mathrm{g})$ según los estadios. 


\section{CONGRESO IBÉRICO DE AGROINGENIERÍA \\ X CONGRESSO IBÉRICO DE AGROENGENHARIA \\ 3 - 6 septiembre 2019, Huesca - España}

Tabla 8. Prueba de rangos múltiples de Duncan del efecto del estadio en el ratio fuerza de retención entre masa $(\mathrm{N} / \mathrm{g})$.

\begin{tabular}{ccccc}
\hline Estadio & Casos & Media & & Grupos Homogéneos \\
\hline 5 & 20 & 2,398 & $X$ & \\
4 & 40 & 3,703 & $X$ & \\
3 & 40 & 5,5495 & $X$ & \\
2 & 20 & 10,9855 & $X$ & \\
1 & 20 & 32,8215 & \multicolumn{2}{c}{$\mathrm{X}$} \\
\hline
\end{tabular}

\subsection{Evolución del porcentaje de derribo}

En un segundo paso se estudió el efecto de los factores frecuencia y semana en el porcentaje de derribo, tabla 9. Ambos factores afectan de forma significativa en el porcentaje de derribo. Las ramas vibradas con la frecuencia mayor $(38 \mathrm{~Hz})$ registraron un porcentaje de derribo significativamente mayor que las vibradas con la frecuencia menor $(18 \mathrm{~Hz})$, figura 8 . En el caso de la frecuencia menor el porcentaje de derribo medio se encontró por debajo de los niveles aceptables para un correcto aclareo, en torno al $10 \%$, después de vibrar 10 segundos.

Tabla 9. Análisis de varianza (suma de cuadrados tipo II) del efecto de los factores frecuencia (38 $\mathrm{Hz}$ y $18 \mathrm{~Hz}$ ) y semana (de 1 a 7 ) en el porcentaje de derribo final de cada rama.

\begin{tabular}{cccccc}
\hline Fuente & Suma de Cuadrados & Gl & Cuadrado Medio & Razón-F & Valor-P \\
\hline EFECTOS PRINCIPALES & & & & & \\
A:Semana & 9798,8 & 6 & 1633,13 & 4,48 & 0,0006 \\
B:Frecuencia (ud) & 47553,2 & 1 & 47553,2 & 130,37 & 0,0000 \\
INTERACCIONES & & & & & \\
AB & 7663,16 & 6 & 1277,19 & 3,50 & 0,0041 \\
RESIDUOS & 27721,2 & 76 & 364,752 & & \\
TOTAL (CORREGIDO) & 93993,1 & 89 & & & \\
\hline
\end{tabular}

Todas las razones-F se basan en el cuadrado medio del error residual

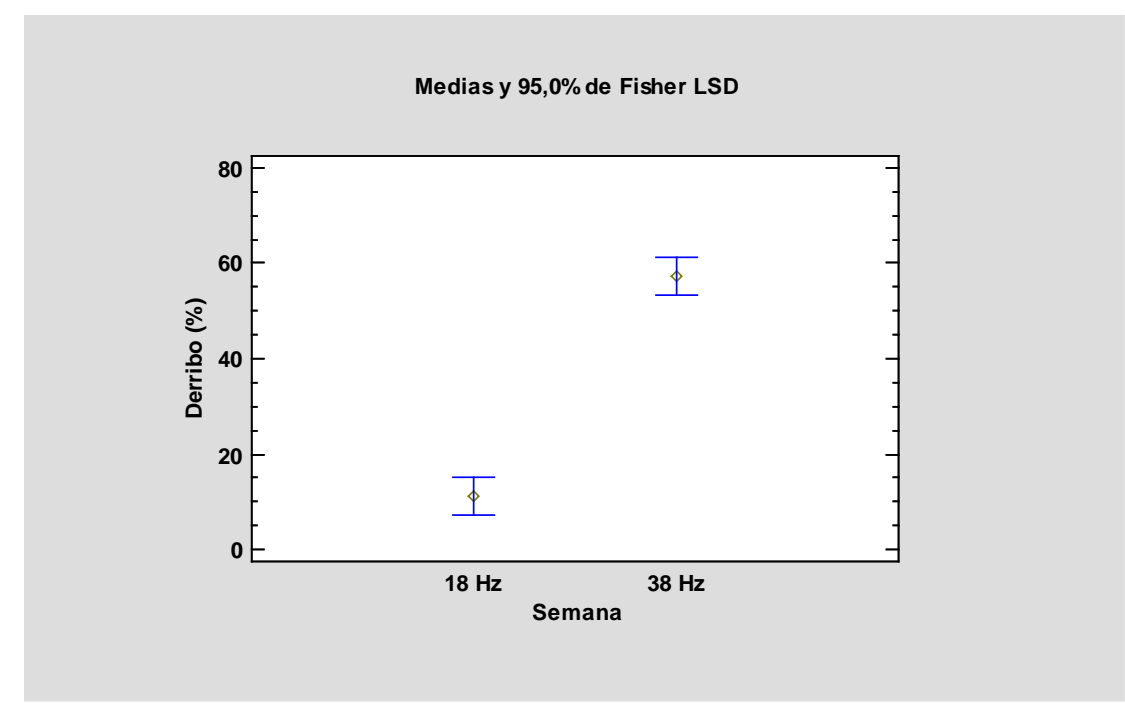

Figura 8. Porcentaje de derribo de los frutos según la frecuencia.

Si se compara la evolución del porcentaje de derribo para la frecuencia mayor $(38 \mathrm{~Hz})$ frente a la evolución del ratio fuerza de retención entre masa se observa que el descenso en el ratio 


\section{CONGRESO IBÉRICO DE AGROINGENIERÍA \\ X CONGRESSO IBÉRICO DE AGROENGENHARIA \\ 3 - 6 septiembre 2019, Huesca - España}

fuerza de retención entre masa se corresponde con una ascenso del porcentaje de derribo, figura 9. Esto sucede hasta el estadio 5 (calibre de $30,8 \mathrm{~mm}$ ) correspondiente con un importante incremento de la masa, que pasa de $8,7 \mathrm{~g}$ de media a $15,5 \mathrm{~g}$ de media.

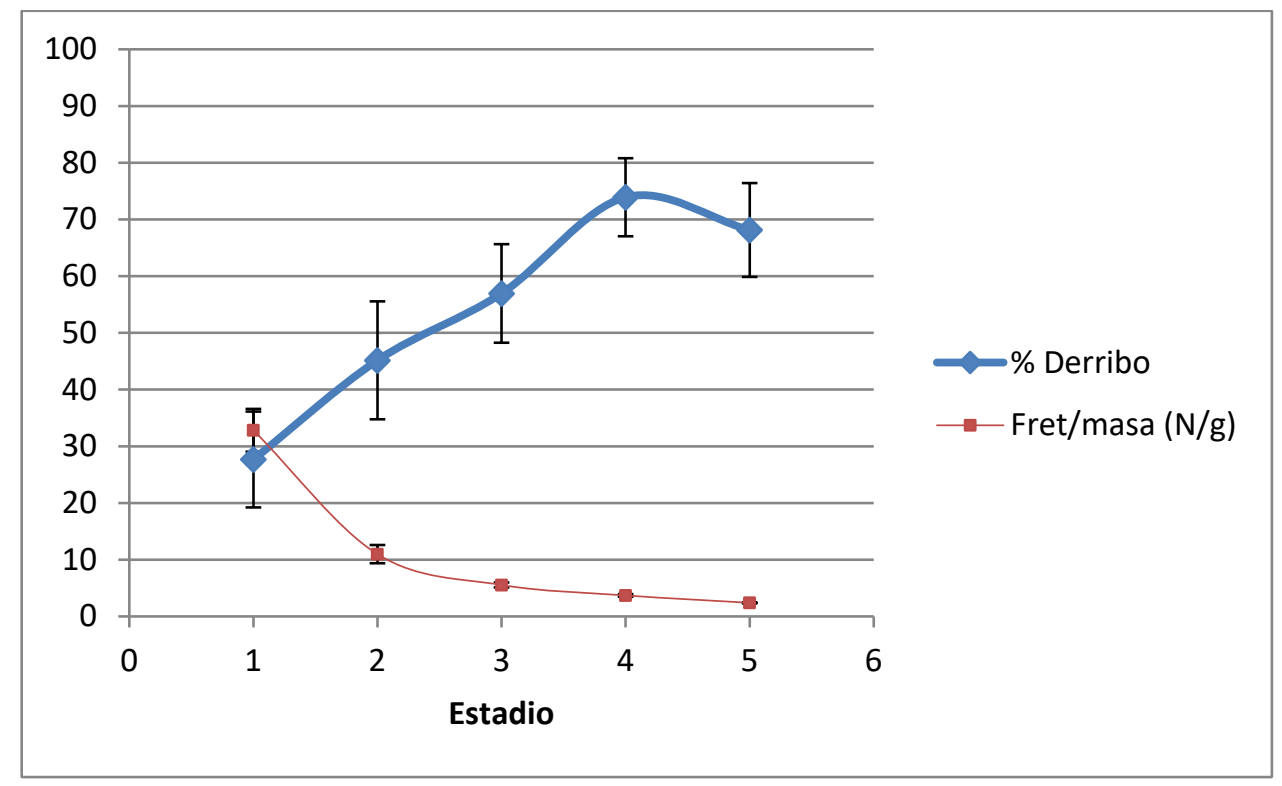

Figura 9. Porcentaje de derribo de los frutos y ratio fuerza de retención entre masa según el estadio (medias y errores típicos).

Posteriormente, se establecieron, a través de las películas de alta velocidad, los tiempos de derribo de los distintos frutos de cada rama y se calcularon los porcentajes de derribo acumulados por cada rama separada frente al tiempo en segundos, para la frecuencia de $38 \mathrm{~Hz}$, figura 10.

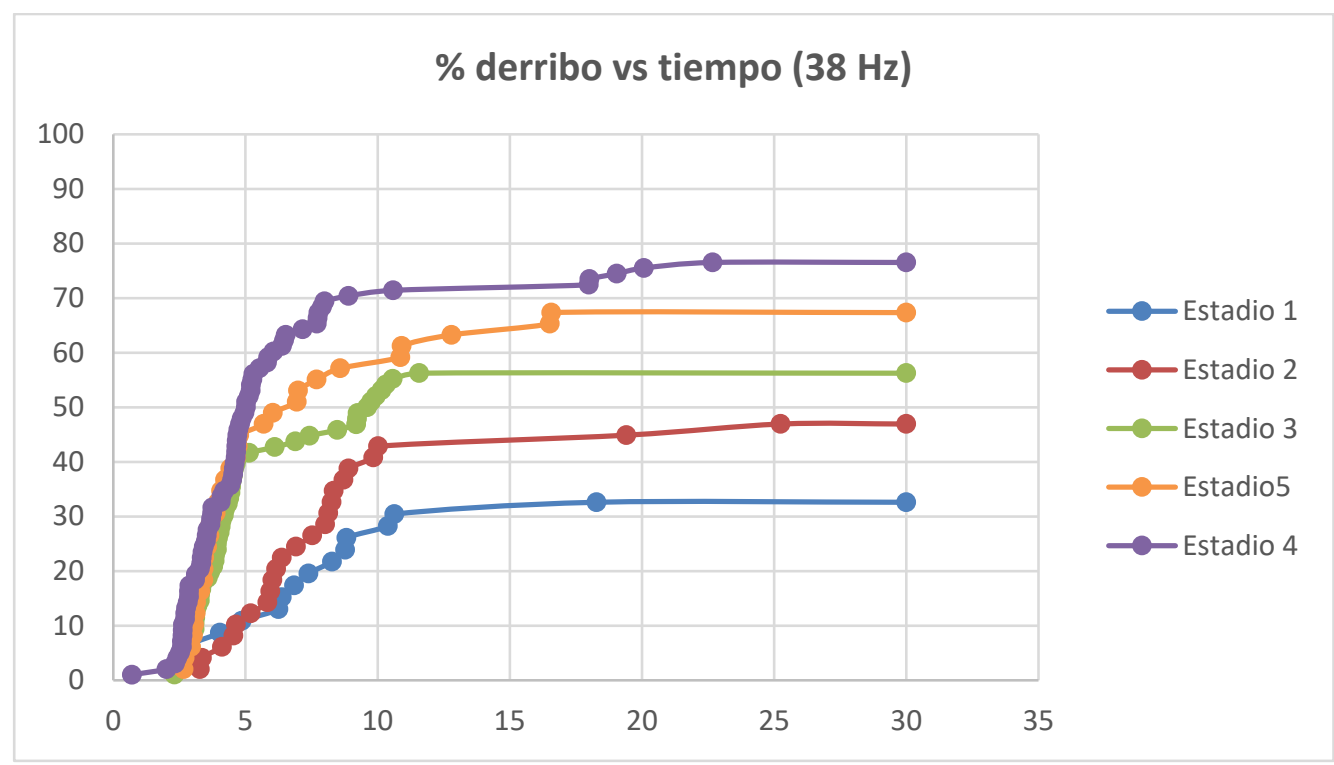

Figura 10. Curvas de evolución del porcentaje de derribo acumulado para los distintos estadios.

Se confirma un porcentaje de derribo creciente según el estadio, a excepción del estadio 5, como ya se había comprobado. Además, se observa un incremento de la pendiente de la curva de derribo según avanza el estadio. 


\section{CONGRESO IBÉRICO DE AGROINGENIERÍA \\ X CONGRESSO IBÉRICO DE AGROENGENHARIA \\ 3-6 septiembre 2019, Huesca - España}

Las curvas de derribo frente al tiempo se ajustan a una curva inversa de $\mathrm{X}$ o de tipo $\mathrm{S}$, con un R2 medio de $84,2 \%$ en el caso de la curva en $\mathrm{S}$ y $92,3 \%$ en el caso de la curva inversa.

Para poder conseguir un porcentaje de derribo aceptable (en torno a $30 \%$ ) se podría vibrar 5 segundos si los frutos tienen un calibre mayor de $20 \mathrm{~mm}$. Sin embargo, si los frutos son menores, el tiempo de vibrado debería ser mayor de $8 \mathrm{~s}$ para diámetros de $14 \mathrm{~mm}$ y más de $10 \mathrm{~s}$ para calibres de $8 \mathrm{~mm}$.

Si consideramos la pendiente en el primer tramo de las curvas vemos como se incrementa según avanza el estadio, tabla 10.

Tabla 10. Valores de la pendiente de la recta de ajuste y el $\mathrm{R}^{2}$ para cada estadio.

\begin{tabular}{ccc}
\hline Estadio & Pendiente primer tramo recto & R2 ajuste recta \\
\hline 1 & 2,98 & 96,54 \\
2 & 5,93 & 96,85 \\
3 & 12,59 & 88,34 \\
4 & 14,72 & 94,19 \\
5 & 20,40 & 97,12 \\
\hline
\end{tabular}

\section{Conclusiones}

Se confirma una evolución ascendente de los valores de calibre, masa y fuerza de retención según evolucionan las semanas de desarrollo del fruto. Sin embargo, se ha comprobado como la selección de las muestras en semanas consecutivas no se correspondía siempre con estados fenológicos diferentes, pues el calibre y la masa no presentaban diferencias significativas.

Considerando los estadios de desarrollo del fruto, el ratio fuerza de retención del fruto entre masa disminuye en los estadios 1, 2 y 3, manteniéndose con valores similares en los estadios 3, 4 y 5 .

Tanto el factor semana, como la frecuencia afectan significativamente al porcentaje de derribo total de los frutos. Las ramas vibradas con una frecuencia de $38 \mathrm{~Hz}$ presentan porcentajes de derribo significativamente mayores que con la frecuencia de $18 \mathrm{~Hz}$. Con la frecuencia de 18 $\mathrm{Hz}$ el porcentaje de derribo de los frutos se considera insuficiente (un valor medio en torno a 10 $\%$ con 10 segundos de vibrado).

$\mathrm{Al}$ evolucionar el desarrollo del fruto (estadio) el porcentaje de derribo se incrementa. Este hecho cambia en el estadio 5, cuando el porcentaje de derribo, con la frecuencia de $38 \mathrm{~Hz}$, parece cambiar su tendencia ascendente. Esto puede ser debido al incremento considerable de la masa del fruto (pasa de un valor medio de $8,7 \mathrm{~g}$ a $15,5 \mathrm{~g}$ ).

Las curvas de porcentaje de derribo frente al tiempo confirman el porcentaje de derribo ascendente segúna avanza el estadio, a excepción del estadio 5.

Las curvas de derrribo frente al tiempo se ajustan a una curva inversa de $\mathrm{X}$ o de tipo $\mathrm{S}$.

La pendiente de la recta del tramo inicial de las curvas de derribo frente a tiempo suben según el estadio.

Para poder derrribar los frutos mediante aclareo mecánico, con la carrera estudiada (30 mm), sería conveniente utilizar la frecuencia de $38 \mathrm{~Hz}$ y no la de $18 \mathrm{~Hz}$.

Los resultados de las curvas de derribo frente al tiempo parecen indicar una relación entre el tiempo de vibrado y el derribo, para cada estadio.

Para poder conseguir un porcentaje de derribo aceptable (en torno a $30 \%$ ) se podría vibrar 5 segundos si los frutos tienen un calibre mayor de $20 \mathrm{~mm}$ (bajo las condiciones de laboratorio del estudio). Sin embargo, si los frutos están en un nivel de desarrollo menor, el tiempo de vibrado debería ser mayor de $8 \mathrm{~s}$ para calibres de $14 \mathrm{~mm}$ y más de $10 \mathrm{~s}$ para calibres de $8 \mathrm{~mm}$ (bajo las considiciones de laboratorio del estudio). 


\section{CONGRESO IBÉRICO DE AGROINGENIERÍA \\ X CONGRESSO IBÉRICO DE AGROENGENHARIA \\ 3 - 6 septiembre 2019, Huesca - España}

\section{Agradecimientos}

Este trabajo ha sido financiado por el Instituto Nacional de Investigación y Tecnología Agraria y Alimentaria (INIA) y fondos FEDER (Proyecto RTA2014-00025-C05-02).

\section{Referencias}

1. Stander, O.P.J and Cronjé P.J.R. Reviewing the Commercial Potential of Hand Thinning in Citrus with a Cost-benefit Analysis of Summer Hand Thinning of 'Nadorcott' Mandarin. HortTechnology. 2016, 26(2), 206-212.

2. Mesejo C., Rosito S., Reig C., Martinez-Fuentes A., Agustí, M. Journal of Plant Growth Regulation. 2012, 31: 186-194.

3. DOI: $10.1007 / \mathrm{s} 00344-011-9230-z$

4. Gonzatto M.P., Böettcher G.N., Schneider L.A., Silveira J.C., Petry H.B., Pedroso de Oliveira R., Schwarz S.F. 3,5,6-trichloro-2-pyridinyloxyacetic acid as effective thinning agent for fruit of ‘Montenegrina' mandarin. Ciência Rural, Santa Maria. 2016, 46(12), 2078-2083.

5. DOI: $10.1590 / 0103-8478$ cr20140057

6. Ouma, G. Fruit thinning with specific reference to citrus species: A review. Agriculture and Biology Journal of North America. 2012, 3(4): 175-191

7. Powell, A.A., Hancock, B.G., Puls, E.E., Helmers Jr., S.G., Brown Jr., M.H. Utilizing

8. mechanical fruit thinning in commercial peach orchards. HortScience. 1975, 10 (2),

9. 142 .

10. Rosa, U.A., Thompson, J.F., Slaughter, D., DeJong, T., Shackel, K., Tiamzon, J., Johnson,

11. S. Feasibility study of using electromagnetic shaker technology for peach

12. thinning. In: Proc. Int. Conf. AgEng2004, Leuven, Belgium. 2004, 12-16 September, pp.

13. 1116-1117

14. Schupp J.R., Baugher, T.A., Miller, S.S., Harsh, R.M., lesser, K.M. Mechanical Thinning of Peach and Apple Trees Reduces Labor Input and Increases Fruit Size. HortTechnology. 2008, 18 (4), 660-670.

15. Miller, S.S., Schupp, J.R., Baugher T.A., Wolford S.D. Performance of Mechanical Thinners for Bloom or Green Fruit Thinning in Peaches. HortScience. 2011, 46(1), 43-51.

16. Seehuber C., Damerow L. and Blanke M.M. Concepts of selective mechanical thinning in fruit tree crops. Acta Horticulturae. 2013, 998: 77-83

17. DOI: $10.17660 /$ ActaHortic.2013.998.8

18. Ortiz, C., Martín-Gorriz, B., Ortí, E. And Torregrosa, A. An approach to mechanical thinning of mandarins.ASABE Annual International. 2016. Meeting162457303.

19. DOI:10.13031/aim.20162457303

20. Torregrosa, A., Orti, E., Soria, E., Balasch, S., Ortiz, C. Size Development of 'Clemenrubi' Mandarin Fruit After Manual And Mechanical Thinning. Eur AgEng Conference, Wageningen (Holanda). 2018, 8-11 de junio

21. Albrigo L.G., Valiente J.I., Beck H.W. Flowering expert system development for a phenology based citrus decision support system. SHS Acta Horticulturae. 2001, 584: VI International Symposium on Computer Modelling in Fruit Research and Orchard Management

22. Ortiz C., Torregrosa T. Determining adequate vibration frequency, amplitude, and time for mechanical harvesting of fresh mandarins. Transactions of the ASABE. 2013, 56(1), 15-22

23. DOI: $10.13031 / 2013.42581$

24. Agustí M., Zaragoza S., Bleiholder H., Buhr 1., Hack H., Klose R., Staus R. Adaptation of the BBCH scale for the description of Citrus fruits' phenological stages. Fruit. 1997, 52(5): 287-295 